\title{
Manuscript
}

"NOTICE: this is the author's version of a work that was accepted for publication in Microelectronics Reliability. Changes resulting from the

publishing process, such as peer review, editing, corrections, structural formatting, and other quality control mechanisms may not be

reflected in this document. Changes may have been made to this work since it was submitted for publication. A definitive version was

subsequently published in Microelectronics Reliability, VOL.55, ISSUES 9-10, August-September 2015 DOI:10.1016/j.microrel.2015.06.084

\section{Charge Induced by Ionizing Radiation Understood as a Disturbance in a Sliding Mode Control of Dielectric Charge}

\author{
Manuel Domínguez-Pumar ${ }^{1}$, Sergi Gorreta ${ }^{1}$, Joan Pons-Nin ${ }^{1}$, Faustino Gómez-Rodríguez ${ }^{2}$, \\ Diego M. González-Castaño ${ }^{2}$ \\ ${ }^{1}$ Micro and Nano Technologies Group, DEE, Universitat Politècnica de Catalunya, Barcelona, Spain \\ ${ }^{2}$ Radiation Physics Laboratory, Universidade de Santiago de Compostela, Spain \\ Corresponding author: Manuel Domínguez-Pumar; e-mail: manuel.dominguez@upc.edu
}

\begin{abstract}
The purpose of this paper is to show that the charge induced by radiation in a dielectric on which a sigmadelta control of dielectric charge is implemented, can be seen as a disturbance in a sliding mode controller. Preliminary experimental results are presented in which a MEMS device is irradiated with $\mathrm{X}$-rays, while the dielectric charge control is continuously being monitored. The charge induced by radiation generates a change in the control bitstream, which is associated to the presence of an external disturbance on the governing control equations.
\end{abstract}

Index Terms - dielectric charge control, sliding mode control, charge trapping, ionizing radiation

\section{INTRODUCTION}

The effects of radiation on semiconductor devices working in harsh environments such as space applications have been extensively studied. The interaction of ionizing radiation, such as $\mathrm{X}$-Rays, gamma-rays and heavy ions, with electronic devices is an issue to be cared of in all missions. Ionizing radiation produces charge trapping in the insulator layers, resulting in undesired drift of the electrical properties of the devices. For instance, this causes changes in the threshold voltage of MOS transistors. In order to avoid this problem shielding strategies, providing reduction of radiation doses, and specific faulttolerant design techniques have been developed and used to improve the reliability of MOS circuits in space.

The study of radiation effects on MEMS devices has received comparatively less attention. However, the interest in this matter is growing, mainly due to the increasing utilization of these technologies in space applications. Many different devices have been irradiated with gamma rays, protons, electrons, X-rays and heavy ions [1]. These studies have been carried out on RF switches, comb drives, accelerometers, etc. It has been observed that at high radiation doses the main failure mechanism is charge trapping in the dielectrics present in the structure. This changes noticeably the electrostatic force and therefore generates undesired drifts of the characteristics of the devices, such as pull-in and pull-out voltages [2]-[5].

Additionally to ionizing radiation, dielectric charging may also be caused by the actuation applied to the device. Usually a complex combination of processes may contribute to the injection of charge into the dielectric, such as trap-assisted tunnelling (TAT) and Schottky emission from the conductors directly into the dielectrics [6]-[10]. This reliability problem has slowed down the introduction of MEMS devices in some applications [11]. Accordingly to its importance, MEMS dielectric charging has received considerable research attention over the past years. In order to mitigate the effects of actuation-induced charging, several open-loop strategies using bipolar actuations have been proposed [11], [12]. The main drawback of these strategies is that they cannot guarantee long-term stability since they cannot adapt to drifts of the charging characteristics of the dielectric layers. In view of this, a family of sigma-delta closed-loop controls has been proposed recently, [13]-[15]. These controls generate adequate sequences of bipolar actuation voltages to keep constant at the desired level the total charge in the dielectric, or what is the same the voltage shift of the of capacitance-voltage, $C-V$, curve of the device.

Recently, it has been shown in [16] that these dielectric charge controllers can partially compensate charge induced by ionizing radiation. In this way, the balance of charge in the dielectric, seen now as a charge reservoir, is determined by three different components:

- charge being induced by the voltage sequence generated by the controller,

- charge being leaked out of the dielectric, and

- charge being induced by radiation.

This means that during an experiment on which a device is being irradiated while it is controlled, the actuation must dynamically adapt to compensate the charge being induced by the radiation so as to keep constant the desired level of charge. This implies that changes in the instantaneous dose rates delivered must generate changes on the output sequences produced by the controllers. This has been indeed shown in [16], where the radiation dose rate pattern of the experiments can be correlated with changes in the output bitstream provided by the control loops.

In this work the charge dynamics will be analyzed using multi-exponential models, [13], [14]. It will be shown that the usual slow time changes in the output sequences of the controllers can be understood as the result of adding a 


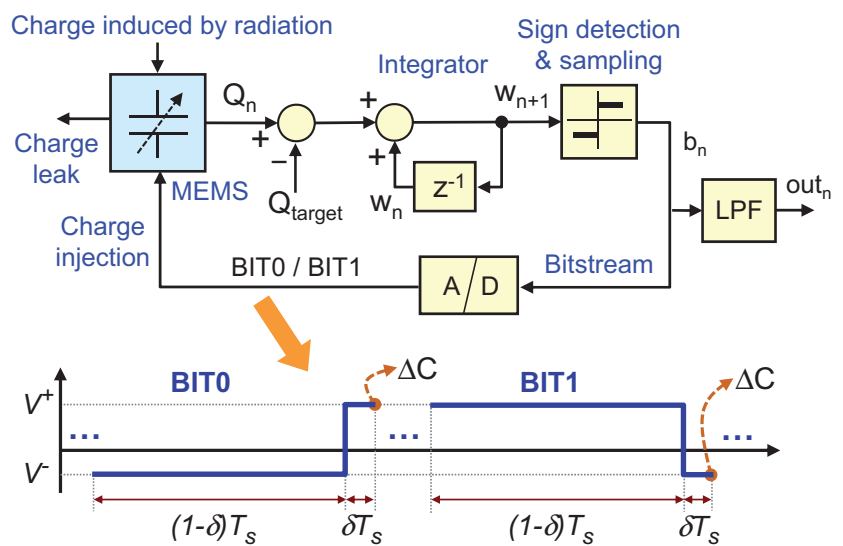

Fig. 1. Top: Second order sigma-delta control of dielectric charge. Bottom: Bipolar voltage waveforms applied by the control scheme. Switching of voltages within each symbol is used to obtain an indirect measurement of the charge accumulated in the dielectric. Real-time information about the charge status is obtained by averaging the output bitstream.

disturbance to the multi-exponential models within the control loop. In particular the addition of a step of charge induction rate, produces a slow time evolution in the output bitstream. In order to study whether this slow behaviour is the only possible, the controllers are analyzed from the point of view of sliding mode controllers. Simulations will show that if the charge induction rates generated by the radiation match the charge induction rates generated by the actuation, a fast response can be obtained. Since this condition is not easy to be fulfilled, it is to be expected that sudden changes in the radiation dose rates generate slow time responses in the output bitstreams. Finally, experimental results will be shown in which a device being controlled by a second order sigma-delta control of charge is irradiated with X-rays, producing the expected slow time response in the bitstream.

\section{Sigma-Delta CONTROL OF DiELECTRIC CHARGING}

The block diagram shown in Figure 1 describes the secondorder sigma-delta control of dielectric charge proposed in [15] and used in this work. It is a sampled-time circuit in which only two voltage values, $V^{+}>0$ and $V^{-}<0$, are used to actuate the device. Concretely, at each sampling period, $T_{S}$, one of the bipolar voltage waveforms, BIT0 or BIT1, also shown in Figure 1 is applied to the MEMS.

BIT0 and BIT1 waveforms produce complementary effects on the dynamics of the dielectric charging. For instance, when the device is working below pull-in (contactless case), applying BIT0s (negative voltage $V^{-}$dominant) increase the amount of positive charge in the dielectric and thus cause horizontal shift of the $C-V$ characteristic towards positive voltages (i.e. the voltage shift, $V_{s h}$, increases). At their turn, BIT1s (positive voltage $V^{+}$dominant) tend to increase the negative charge, and thus to decrease $V_{s h}$. This behaviour is consistent with the experimental results obtained with RF MEMS switches reported recently [17].

Additionally, BIT0 and BIT1 waveforms allow indirect sensing of the total amount of dielectric charge. This is made from a "quasi-differential" capacitance value, defined as $\Delta C:=C\left(V^{+}\right)-C\left(V^{-}\right)$, obtained from two capacitance measurements, $C\left(V^{+}\right)$and $C\left(V^{-}\right)$, performed at times $(1-$ $\delta) T_{S}$ and $T_{S}$ within each waveform. Under some regularity conditions of the $C-V$ and assuming that $T_{S}$ is far below the time constants of the charge dynamics, $\Delta C$ has been shown to be an affine function of $V_{s h}$, and therefore of the total charge in the dielectric [14]. This implies that an indirect measurement of the dielectric charge, $Q_{n}$, is available at each sampling time $n T_{S}$. This kind of measurement is insensitive to vertical shifts of the $C-V$ that can be caused by charge inhomogeneity [18] or by changes in environmental factors such as temperature or humidity [19], [20]. Note that charge sensing is built-in with device actuation, so it is less invasive than other common methods used to detect the total charge, such as measuring $V_{s h}$ as the displacement of the pull-in voltage (which involves applying voltage stress to close the device and thus takes time and dramatically alters the charge status) or performing shortrange $C-V$ measurements to detect the displacement of the minimum of the curve [21].

In the sigma-delta controls proposed in [14] and [15], the dielectric is seen as a leaky charge integrator working under two competing mechanisms: charge being injected by the actuation waveforms BIT0 and BIT1 and charge escaping the traps in the material. The sigma-delta loops generate sequences of BIT0 and BIT1 waveforms (output bitstream) that compensate in average the charge being leaked out of the dielectric, thus allowing to reach and maintain a given value of total dielectric charge $Q_{n}=Q_{\text {target }}$. In the firstorder case, the decision of whether BIT0 or BIT1 is to be applied in the next sampling period is taken directly from the sign of $Q_{n}-Q_{\text {target }}$, in practice evaluated as $\Delta C_{n}-\Delta C_{\text {target }}$. In the second order control used here, the decision is taken from the sign provided by the time integral of $Q_{n}-Q_{\text {target }}$, see Figure 1. This integrator improves noise rejection at low frequencies, by providing a second-order quantization noise shaping. Finally, real-time information about the dielectric charge status is obtained by low-pass filtering (or averaging) the output bitstream $b_{n}$ provided by the control loop.

\section{A. Multi-exponential time-varying charge model}

Let us now model the charge control system described above. To this effect, we consider first a multi-exponential time-varying dielectric charging model to describe the dynamics of the total charge in the device:

$$
\dot{x}(t)= \begin{cases}A_{1} x(t)+B_{1}, & v(t)=V^{+} \\ A_{0} x(t)+B_{0}, & v(t)=V^{-}\end{cases}
$$

where $A_{i} \in \mathbb{R}^{n \times n}, B_{i} \in \mathbb{R}^{n}, i=\{0,1\}$, and $v(t) \in \mathbb{R}$ is the control voltage applied to the device: either a constant positive or negative bias: $V^{+}>0$, or $V^{-}<0$. We assume that matrices $A_{i}$ are stable with real eigenvalues. As discussed above, in the contactless case we have that $B_{0} \geq 0$ and $B_{1} \leq 0$, i.e, for positive voltage negative charge is injected, and positive charge otherwise. At the moment of switching between voltages the state vector, namely $x(t)$, is continuous. The output of the 
system in our case is:

$$
Q(t)=c^{T} x(t)
$$

where $c=(1, \cdots, 1)^{T} \in \mathbb{R}^{n}$ and $Q(t) \in \mathbb{R}$ represents the net charge in the device. This model can be seen therefore as a SISO hybrid system.

\section{B. Description of the actuation waveforms}

The two actuation waveforms BIT0 and BIT1 (see Figure 1) used to implement the indirect sensing of the total charge and the necessary actuation to keep the desired level of charge can be described as follows:

$$
v_{\text {BIT0 }}(t)= \begin{cases}V^{-}, & t \in\left[0,(1-\delta) T_{S}\right) \\ V^{+}, & t \in\left[(1-\delta) T_{S}, T_{S}\right) \\ 0, & t \notin\left[0, T_{S}\right)\end{cases}
$$

and:

$$
v_{\mathrm{BIT} 1}(t)= \begin{cases}V^{+}, & t \in\left[0,(1-\delta) T_{S}\right) \\ V^{-}, & t \in\left[(1-\delta) T_{S}, T_{S}\right) \\ 0, & t \notin\left[0, T_{S}\right)\end{cases}
$$

With the above definitions, a first-order sigma-delta control of charge is then:

$$
\begin{aligned}
\dot{x}(t) & =A_{v(t)} x(t)+B_{v(t)} \\
v(t) & =\sum_{n} \frac{1}{2}\left(1+b_{n}\right) v_{\mathrm{BIT} 1}\left(t-n T_{S}\right) \\
& +\sum_{n} \frac{1}{2}\left(1-b_{n}\right) v_{\mathrm{BIT} 0}\left(t-n T_{S}\right) \\
b_{n} & =\operatorname{sgn}\left(c^{T} x\left(n T_{S}\right)-Q_{\text {target }}\right)
\end{aligned}
$$

where $A_{v(t)}=A_{1}, B_{v(t)}=B_{1}$ for $v(t)=V^{+}$and $A_{v(t)}=$ $A_{0}, B_{v(t)}=B_{0}$ for $v(t)=V^{-}$. $Q_{\text {target }}$ is the desired level of total charge.

In the case of a second order control, the integrator provides an additional state variable:

$$
w_{n+1}=w_{n}+\Delta Q_{n}
$$

where $\Delta Q_{n}=c^{T} x\left(n T_{S}\right)-Q_{\text {target }}$, and then the generated bitstream is $b_{n}=\operatorname{sgn}\left(w_{n}+\Delta Q_{n}\right)$.

\section{Average system: switching within symbols BITO and BIT1}

The voltage waveforms used by the sigma-delta controls present a two level hierarchy:

- First level: the voltage switching within each BIT0 and BIT1 symbol. This switching is time-programmed and basically depends on the values of the sampling period $T_{S}$ and of the parameter $\delta$.

- Second level: the switching between BIT0 and BIT1 symbols. This switching depends on the instantaneous value of the total charge at the sampling periods $n T_{S}$. This second level, therefore is dependent on the sampled state vector, $x$.
In order to analyze the system we will proceed by obtaining an equivalent system that will take into account the first level of switching, under what is usually called 'infinite sampling approximation'. This implies a sampling period at least one order of magnitude below the shortest time constant in the affine models (1). The main purpose of this analysis is to obtain two time-invariant models that represent the time evolution of the state variables under a constant application of either BIT0 or BIT1 symbols.

We will consider that a convex combination of systems $\left\{A_{0}, B_{0}\right\}$ and $\left\{A_{1}, B_{1}\right\}$ is obtained when switching fast enough between both systems:

- Constant application of BIT0 symbols: during a BIT0, $(1-\delta)$ percentage of the time $V^{-}$is applied and $V^{+}$ during the remaining time.

- Constant application of BIT1 symbols: we have the complementary situation.

Therefore, the first-order sigma-delta control of charge can be described as, in the limit $T_{S} \rightarrow 0$ :

$$
\dot{x}= \begin{cases}A_{b_{1}} x+B_{b_{1}}, & \sigma>0 \\ A_{b_{0}} x+B_{b_{0}}, & \sigma<0\end{cases}
$$

with $\sigma(x)=c^{T} x(t)-Q_{\text {target }}$ and:

$$
\begin{aligned}
& A_{b_{1}}=(1-\delta) A_{1}+\delta A_{0} \\
& B_{b_{1}}=(1-\delta) B_{1}+\delta B_{0} \\
& A_{b_{0}}=\delta A_{1}+(1-\delta) A_{0} \\
& B_{b_{0}}=\delta B_{1}+(1-\delta) B_{0}
\end{aligned}
$$

With expression (7) it is possible to 'forget' the continuous voltage switching due to the indirect measurement of the dielectric charge within each sampling period.

\section{ANALYSIS OF THE EFFECT OF THE CHARGE INDUCED BY IONIZING RADIATION ON THE CONTROL LOOP}

\section{A. Sliding mode control}

Expression (7) can be seen as a particular case of the general expression for a time-varying system on which the time evolution depends on the instantaneous value of a function of the state vector. In our case, this function is the value of the total charge, i.e., $\sigma(x)=c^{T} x-Q_{\text {target }}$ which is called the control surface.

$$
\dot{x}(t)=f(\sigma(x))
$$

On the other hand, $f(\sigma(x))$ is a discontinuous function of the state vector, $x$, since the time evolution of the state variables is determined by the sign of $\sigma(x)$. This is something to be expected because the sigma-delta control applies a BIT1 symbol when the charge is above the desired level, and a BIT0 otherwise. It may happen therefore what has been called in the literature 'fast switching regime' in relay feedback systems, or also sliding regime. In this regime, the control surface, $\sigma(x)=0$, is attractive and the dynamical system follows a trajectory that, as a first order approximation, lies precisely on the discontinuity of the model (the control surface $\sigma=0$ ). This means that the control scheme applies a feedback signal 
$\left(b_{n}\right)$ such that in average it moves the system on the control surface $\sigma=0$.

Assuming a sliding region, we have that around a neighbourhood of any point on the control surface belonging to the sliding region, the trajectory followed by (9) will bring the system again onto the surface, i.e., it is an attractive set.

In order to analyze the effect of the charge induced by radiation on the dielectric charge control it is preferable to express system (7) as:

$$
\dot{x}=\frac{1}{2}\left(A_{b_{1}}+A_{b_{0}}\right) x+\frac{1}{2}\left(B_{b_{1}}+B_{b_{0}}\right)+B(x) u
$$

where:

$$
B(x)=\frac{1}{2}\left(A_{b_{1}}-A_{b_{0}}\right) x+\frac{1}{2}\left(B_{b_{1}}-B_{b_{0}}\right)
$$

and $u=\operatorname{sgn}(\sigma(x))$. Signal $u$ may be seen as a feedback control signal applied by the control to keep, under some conditions, the trajectory of the system on the control surface $\sigma(x)=0$. Assuming that the conditions for a sliding regime are met, the equivalent control will be the one ensuring $\dot{\sigma}(x)=c^{T} \dot{x}=0$, if the initial point of the trajectory, $x(0)$ lies on the control surface. We will see in the next section that the charge induced by radiation may be seen as a disturbance on the system (7) affecting the equivalent control generated, as well as the trajectory of the system on the control surface.

\section{B. Charge generation as an external disturbance in the control loop}

In the experimental section below, a first preliminary experiment in which a device has been irradiated with X-rays is presented. Auger and photoelectric effects are the main absorption mechanisms of X-rays while the Compton effects dominates in the range of high photon energies such as in the case of $\gamma$-radiation [2]. The sign of the induced charge in the dielectric layer depends on the final balance between the Inelastic Mean Free Path between the ejected and injected electrons.

On the other hand, in sliding mode controllers, model uncertainties and external disturbances are usually represented by a vector $\phi(x, t) \in \mathbb{R}^{n}$ such that equation (7) in our case takes the form:

$$
\dot{x}=\frac{1}{2}\left(A_{b_{1}}+A_{b_{0}}\right) x+\frac{1}{2}\left(B_{b_{1}}+B_{b_{0}}\right)+B(x) u+\phi(x, t)
$$

In order to qualitatively analyze the effect on the control loop of the charge induced by radiation, simulations have been made on which a constant disturbance is added to the time evolution of the system, as described by (12). As it is well known [22], disturbances can be decomposed in a matched, $\phi_{M}(x, t)$, and a mismatched, $\phi_{U}(x, t)$ component, so that: $\phi(x, t)=\phi_{M}(x, t)+\phi_{U}(x, t)$. The matched component lies inside the space spanned by the column vector $B(x)$, whereas the mismatched component is defined as the component lying on the orthogonal vector space, i.e., $B^{\perp}(x)$. The main difference between the effects of a matched and a mismatched
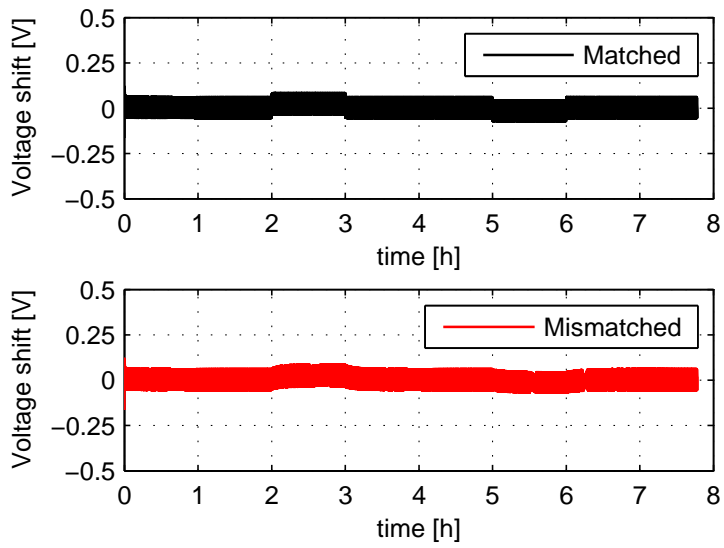

Fig. 2. Comparison between the simulations of a control on which during two time intervals $(2 h \leq t \leq 3 h$ and $5 h \leq t \leq 6 h)$ charge is being generated due to ionizing radiation. Top: when the generated charge is a matched disturbance. Bottom: mismatched disturbance case.

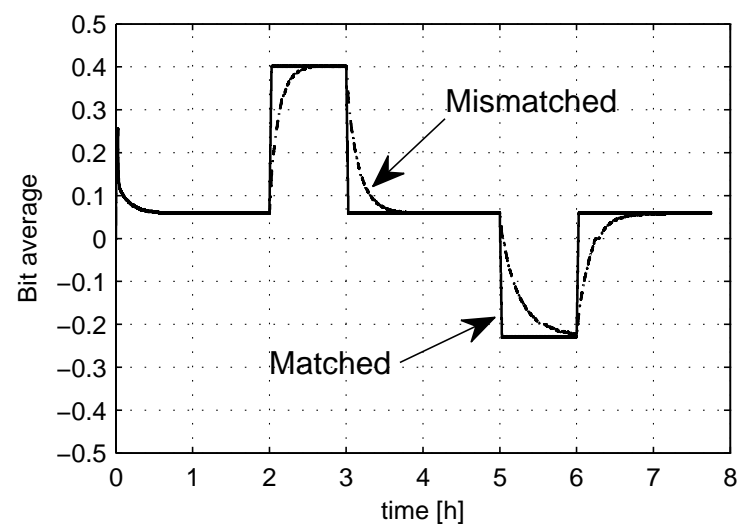

Fig. 3. Comparison between the bitstreams obtained in the simulations of Figure 2.

disturbance can be observed in Figures 2 and 3, in which the charging model obtained in [23, Table 2] has been used.

Figures 2 and 3 show the simulation results of an experiment in which the charge induced by radiation is a matched, or mismatched, disturbance (Figure 2 top and bottom graphs respectively). The simulated equations are those of (7), assuming a sampling clock of $10 \mathrm{~Hz}$. The corresponding average control bitstreams, can be observed in Figure 3. Two different constant generation rate vectors have been used during the simulation, $\phi(x, t)=\phi_{i}, i=0,1$. With the first one, $2 h \leq t \leq 3 h$, the net charge induced by radiation has been positive, whereas in a second radiation pulse, $5 h \leq t \leq 6 h$, a negative generation has been introduced. The main qualitative difference in the response times of the bitstream is that in the matched disturbance case, the bitstream response is almost immediate, whereas in the mismatched case, a slow time evolution is obtained, as expected from a mismatched disturbance. This result qualitatively explains that the control bitstreams of the experiment on which a device was irradiated with X-rays is compatible with a mismatched disturbance due to the charge generated by radiation. 

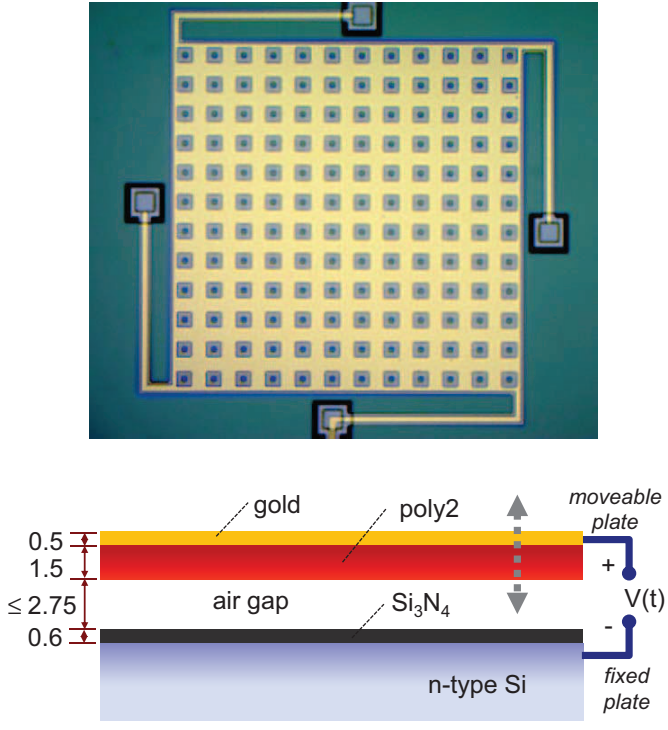

Fig. 4. Top-view photograph and vertical cross section of the capacitive MEMS used in the experiments. All distances are in microns.

\section{EXPERIMENTAL RESULTS}

This section presents a preliminary experiment in which a MEMS has been irradiated with X-rays, while a secondorder sigma-delta control of charge was working at the same time on the device to set a target voltage shift $V_{s h}=-0.5 \mathrm{~V}$. The device is the parallel-plate structure shown in Figure 4. The upper-moveable plate is a suspended stack of polysilicon and gold layers of $360 \times 360 \mu m^{2}$ area. A $0.6 \mu$ m thick $\mathrm{Si}_{3} \mathrm{~N}_{4}$ layer is deposited on top of the silicon substrate, which acts as the bottom plate. In the rest state (zero voltage applied and zero net charge trapped in the dielectric), the air gap between the moveable plate and the dielectric is $2.75 \mu \mathrm{m}$ and the pull-in voltage is $24 \mathrm{~V}$. This device, fabricated with standard PolyMUMPS technology, is from the same batch as the ones used in [14], [15].

A schematic of the measurement set-up can be seen in Figure 5. As commented above, in the sigma-delta controls used an indirect measurement of the total dielectric charge is obtained through quasi differential capacitance measurements. To this effect, a precision LCR meter has been programmed to implement the interface with the MEMS: actuation with BIT0 and BIT1 voltage waveforms and capacitance measurements at the end of each voltage application. In a first order controller it is only necessary to know whether the instantaneous charge is above or below the target level, $Q_{\text {target }}$. To implement a second order controller, though, it is necessary to integrate numerically the difference between the current charge and the target level, $Q_{n}-Q_{\text {target }}$ (in practice, $\Delta C_{n}-\Delta C_{\text {target }}$ ). An advantage of second order controllers is that they do not present the typical plateaus of first-order controllers if low sampling frequencies are used. Additionally, they provide second order quantization noise shaping.

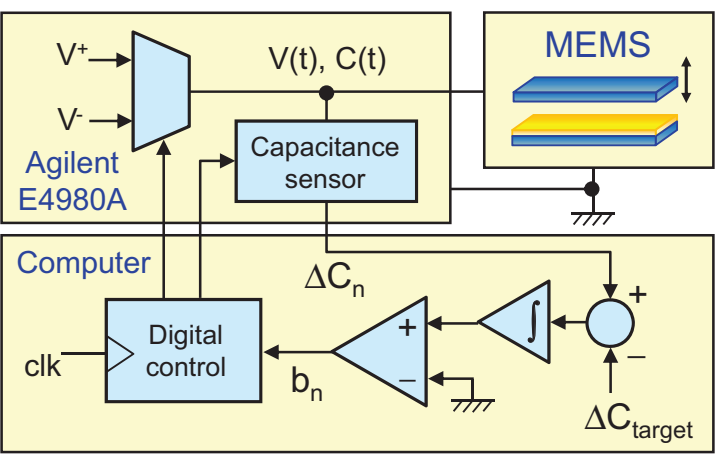

Fig. 5. Experimental set-up. The E4980A impedance analyzer is used to provide the actuation waveforms BIT0 and BIT1 and to sample the capacitance of the device while applying a certain actuation voltage $\left(V^{+}\right.$or $\left.V^{-}\right)$.
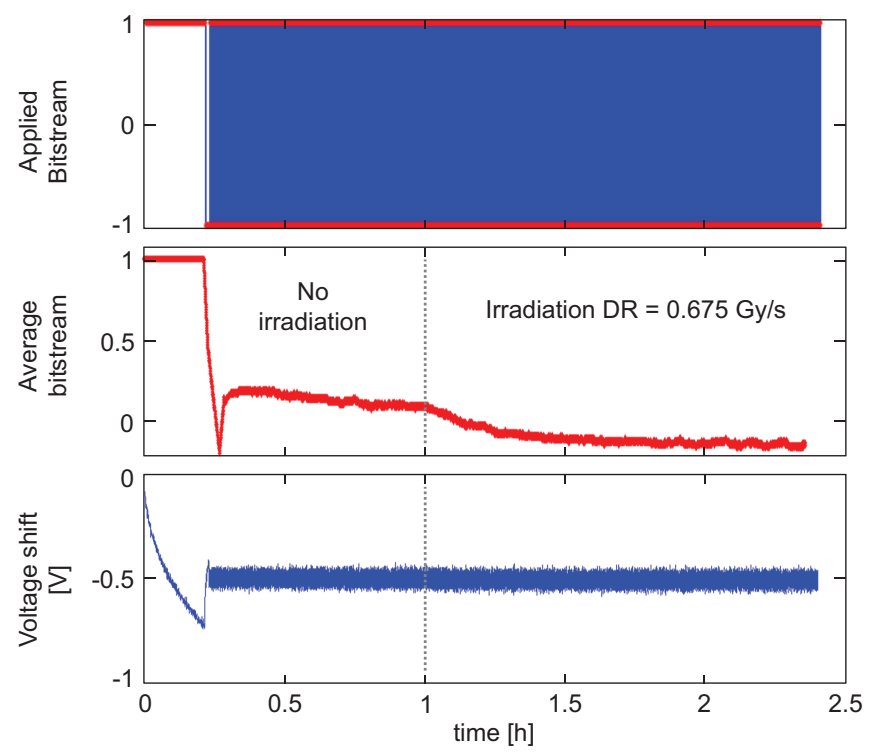

Fig. 6. Experiment consisting on the irradiation of a device with X-rays at a dose rate of $0.675 \mathrm{~Gy} / \mathrm{s}$, while a second-order sigma-delta control of charge sets a target voltage shift of $-0.5 \mathrm{~V}$. The irradiation begins at $t=1 \mathrm{~h}$ and stops at the end of the experiment. Top: applied bitstream. Middle: average bitstream. Bottom: voltage shift as a function of time during the experiment.

The MEMS under test was irradiated by a $50 \mathrm{KV}$ X-ray beam delivered by an Oxford Instruments Neptune tube, with added filtration (window $127 \mu \mathrm{m}$ Beryllium). The experiment was divided in two steps. In the first step the controlled device is not irradiated and the dielectric charge control sets a target voltage shift $V_{s h}=-0.5 \mathrm{~V}$. In the second step, after one hour, a constant dose rate of $0.675 \mathrm{~Gy} / \mathrm{s}$ was delivered to the device, till the end of the experiment.

Figure 6 top graph presents the bitstream applied during the experiment. As it can be observed, during the first 15 minutes only BIT1 symbols were applied. In sliding mode control this phase is called 'reaching phase' and it implies that the control surface has not yet been reached and therefore the actuation being a applied to the device is constant. In our case the control surface is $V_{\mathrm{sh}}=-0.5 \mathrm{~V}$ and since this implies a total negative charge, the excitation is BIT1, consistent with the fact that negative charge must be injected into the dielectric. The slight 
overshoot is due to the presence of the numerical integrator in the control loop. Once the control surface has been reached, a sliding regime is established. In our case this is clearly perceived since there is a continuous switching in the bitstream (see Figure 6 top). Approximately at $t=15 \mathrm{~min}$, the bitstream begins to switch very fast and the voltage shift of the device is kept around its target value. This fast switching regime is also perceived in the voltage shift signal (Figure 6 bottom). The amplitude of the movement around the target value depends on the dynamics of the charge and the sampling frequency. Higher sampling frequencies tend to reduce this error.

The control bitstream can be filtered by averaging (see Figure 6 middle). This average signal provides information about the real-time dynamics of the charge within the dielectric. In sliding mode controllers this is seen as the equivalent control that it is necessary to introduce to continue sliding on the control surface. It must be observed that from the moment the device begins to be irradiated there is a change in the applied control, consistent with a mismatched disturbance and a generation of negative charge due to the X-ray irradiation. Since the bitstream must decrease, i.e. adding more BIT0 symbols, this means that it is necessary to inject more positive charge to keep the desired level of total charge. From this, it can be concluded that the charge being induced by radiation in the dielectric surface is negative.

\section{CONCLUSIONS}

A preliminary experiment has been presented in which a device has been irradiated with X-rays while a second-order sigma-delta control of charge sets a target voltage shift on the device. The change observed in the control bitstream, understood as equivalent control of a sliding mode controller, is compatible with the effect on the system dynamics when a mismatched external disturbance generating a net negative charge is taken into account.

\section{ACKNOWLEDGEMENTS}

This work was supported in part by the Spanish Ministry MINECO under Project TEC2013-48102-C2-1-P.

\section{REFERENCES}

[1] S. T. Patton, A. J. Frasca, J. W. Talnagi, D. J. Hyman, B. S. Phillips, J. G. Jones, R. A. Vaia, and A. A. Voevodin, "Effect of space radiation on the leakage current of MEMS insulators," IEEE Trans. on Nuclear Science, vol. 60, no. 4, pp. 3074 - 3083, 2013.

[2] V. Theonas, M. Exarchos, G. Papaioannou, and G. Konstantinidis, "RF MEMS dielectric sensitivity to electromagnetic radiation," Sensors and Actuators A: Physical, vol. 132, no. 1, pp. 25 - 33, 2006.

[3] H. R. Shea, "Radiation sensitivity of microelectromechanical system devices," Journal of Micro/Nanolithography, MEMS and MOEMS, vol. 8, no. 3, pp. 031 303-031 303-11, 2009.

[4] - "Effects of radiation on MEMS," in Proc. of SPIE Reliability, Packaging, Testing, and Characterization of MEMS/MOEMS and Nanodevices X, vol. 7928, 2011, p. 13.

[5] A. Hartzell, M. Da Silva, and H. Shea, "In-use failures," in MEMS Reliability, ser. MEMS Reference Shelf. Springer US, 2011, pp. 85177.
[6] S. M. Sze and K. K. Ng, Physics of Semiconductor Devices. Third Edition. Wiley, 2007.

[7] M. Razeghi, Fundamentals of Solid State Engineering. Second Edition. Springer, 2006.

[8] A. Gehring and S. Selberherr, "Modeling of tunneling current and gate dielectric reliability for non volatile memory devices," Device and Materials Reliability, IEEE Trans. on, vol. 4, no. 3, pp. 306-319, 2004.

[9] A. Jain, S. Palit, and M. A. Alam, "A physics-based predictive modelling framework for dielectric charging and creep in RF MEMS capacitive switches and varactors," Microelectromechanical Systems, Journal of, vol. 21, no. 2, pp. 420-430, 2012.

[10] A. Koszewski, F. Souchon, C. Dieppedale, D. Bloch, and T. Ouisse, "Physical model of dielectric charging in MEMS," Journal of Micromechanics and Microengineering, vol. 23, no. 4, p. 045019, 2013.

[11] W. Spengen, "Capacitive RF MEMS switch dielectric charging and reliability: a critical review with recommendations," Journal of Micromechanics and Microengineering, vol. 22, 2012.

[12] T. Ikehashi, T. Miyazaki, H. Yamazaki, A. Suzuki, E. Ogawa, S. Miyano, T. Saito, T. Ohguro, T. Miyagi, Y. Sugizaki, N. Otsuka, H. Shibata, and Y. Toyoshima, "An RF MEMS variable capacitor with intelligent bipolar actuation," in IEEE International Solid-State Circuits Conference (ISSCC 2008), Feb 3-7, 2008, pp. 581-583.

[13] E. Blokhina, S. Gorreta, D. Lopez, D. Molinero, O. Feely, J. Pons-Nin, and M. Dominguez-Pumar, "Dielectric charge control in electrostatic MEMS positioners / varactors," Microelectromechanical Systems, Journal of, vol. 21, pp. 559-573, 2012.

[14] S. Gorreta, J. Pons-Nin, E. Blokhina, O. Feely, and M. DominguezPumar, "Delta-sigma control of dielectric charge for contactless capacitive MEMS," Microelectromechanical Systems, Journal of, vol. 23, no. 4, pp. 829-841, Aug 2014.

[15] S. Gorreta, J. Pons-Nin, E. Blokhina, and M. Domínguez-Pumar, "A second order delta-sigma control of dielectric charge for contactless capacitive MEMS," Microelectromechanical Systems, Journal of, vol. 24, no. 2, pp. $259-261,2015$.

[16] M. Dominguez-Pumar, S. Gorreta, J. Pons-Nin, F. Gomez-Rodriguez, D. M. Gonzalez-Castaño, and M. Muschitiello, "Closed-loop compensation of dielectric charge induced by ionizing radiation," Accepted for publication in Microelectromechanical Systems, Journal of, 2015. [Online]. Available: http://ieeexplore.ieee.org/stamp/stamp. jsp?tp=\&arnumber $=7109101 \&$ isnumber $=4357932$

[17] L. Michalas, M. Koutsureli, and G. Papaioannou, "Probing contacless injection dielectric charging in RF MEMS capacitive switches," Electronics Letters, vol. 50, no. 10, pp. 766 - 768, 2014.

[18] X. Rottenberg, I. De Wolf, B. K. J. C. Nauwelaers, W. De Raedt, and H. A. C. Tilmans, "Analytical model of the DC actuation of electrostatic MEMS devices with distributed dielectric charging and nonplanar electrodes," vol. 16, pp. 1243-1253, 2007.

[19] R. W. Herfst, P. G. Steeneken, and J. Schmitz, "Time and voltage dependence of dielectric charging in RF MEMS capacitive switches," in IEEE 45th Annual International Reliability Physics Symposium, Phoenix, 2007, pp. 417-421.

[20] M. Kouteoureli and G. Papaioannou, "The discharge current through the dielectric film in MEMS capacitive switches," in Proc. of the 6th European Microwave Integrated Circuits Conf., 2011, pp. 133-136.

[21] R. W. Herfst, P. G. Steeneken, H. G. A. Huizing, and J. Schmitz, "Centershift method for the characterization of dielectric charging in RF MEMS capacitive switches," IEEE Trans. on Semiconductor Manufacturing, vol. 21, pp. 148-153, 2008.

[22] Y. Shtessel, C. Edwards, L. Fridman, and A. Levant, Sliding Mode Control and Observation. Birkhauser, 2014.

[23] M. Dominguez-Pumar, S. Gorreta, J. Pons-Nin, E. Blokhina, P. Giounanlis, and O. Feely, "Real-time characterization of dielectric charging in contactless capacitive MEMS," Analog Integrated Circuits and Signal Processing, vol. 82, no. 3, pp. 559-569, 2015. 